Mathematical Research Letters 3, 77-91 (1996)

\title{
DISPERSIVE SMOOTHING FOR SCHRÖDINGER EQUATIONS
}

\author{
LeV Kapitanski And Yuri SAFAROV
}

The phenomenon of the global (in time) dispersive smoothing for the "free" Schrödinger evolution can be described as follows: For any distribution $f$ of compact support, the solution $\psi(t, x)$ of the Cauchy problem $\left(\frac{1}{i} \frac{\partial}{\partial t}-\Delta\right) \psi(t, x)=0, t>0, \psi(0, x)=f(x), x \in \mathbb{R}^{n}$, is infinitely differentiable with respect to $t$ and $x$, when $t>0$ and $x \in \mathbb{R}^{n}$. This is equivalent to saying that the corresponding fundamental solution ( $=$ the solution $S_{0}(t, x, y)$ of the initial value problem with $\left.f(x)=\delta(x-y)\right)$ is infinitely differentiable with respect to $t, x$ and $y$, when $t>0$. And we have, indeed, $S_{0}(t, x, y)=e^{-i n \frac{\pi}{4}}(4 \pi t)^{-\frac{n}{2}} \exp \left\{i|x-y|^{2} / 4 t\right\}$, with the only singularity at $t=0$.

One would expect that dispersive smoothing should survive "small" perturbations of the free Hamiltonian $\mathcal{H}_{0}=-\Delta$. The problem is to determine what perturbations are "small".

The case when the perturbed Hamiltonian has the form $\mathcal{H}=\mathcal{H}_{0}+V$ with a potential $V=V(x)$, has been examined in [Ze], [OF], [Ki], [CFKS]. The dispersive smoothing takes place, for example, if the potential is infinitely differentiable, and it and all its derivatives are bounded, [Ze], $[\mathrm{OF}]$. On the other hand, if $V(x)$ grows quadratically or faster at infinity, then the singularities may resurrect, as the example of the quantum harmonic oscillator and Mehler's formula show ([Ze], [We], [CFKS], $[\mathrm{MF}]$ ).

If the perturbation affects the metric of the space, i.e., if $\mathcal{H}_{0}=-\Delta$ is replaced by $\mathcal{H}=-\sum_{j, k=1}^{n} \frac{\partial}{\partial x_{j}} a^{j, k}(x) \frac{\partial}{\partial x_{k}}$, the problem apparently becomes more subtle. Practically no information on global dispersive smoothing

Received July 27, 1995.

Both authors are on leave from the St. Petersburg Branch of Steklov Mathematical Institute, St. Petersburg, Russia

The second author was supported by the Engineering and Physical Sciences Research Council, Great Britain, Grant B/93/AF/1559. 
in that case has been available until recently. At present, however, the situation has changed. In their very interesting paper [CKS], W. Craig, T. Kappeler and W. Strauss prove, in particular, a result that we will now describe.

Assume that the coefficients $a^{j, k}(x)$ of $\mathcal{H}$ are real and $C^{\infty}$, the matrix $\left(a^{j, k}\right)$ is symmetric and positive-definite, and $\left|a^{j, k}(x)-\delta^{j k}\right| \rightarrow 0$ sufficiently fast as $|x| \rightarrow \infty$. It will be convenient to view the principal symbol $H(x, \xi):=\sum_{j, k=1}^{n} a^{j, k}(x) \xi_{j} \xi_{k}$ of the operator $\mathcal{H}$ as the classical Hamiltonian and denote by $(q(s ; x, \xi), p(s ; x, \xi))$ the corresponding Hamiltonian trajectory emanating from the point $(x, \xi)$ of the phase space $\mathbb{R}^{2 n}=T^{*}\left(\mathbb{R}^{n}\right)$. The point $(x, \xi)$ is said to be not trapped forwards (respectively, backwards) by the bicharacteristic flow if $|q(s ; x, \xi)| \rightarrow+\infty$ as $s \rightarrow+\infty$ (respectively, $s \rightarrow-\infty)$. Let $S(t, x, y)$ denote the fundamental solution corresponding to $\mathcal{H}$, i.e., the solution of the initial problem

$$
\begin{aligned}
\frac{1}{i} \frac{\partial}{\partial t} S(t, x, y)+\sum_{j, k=1}^{n} \frac{\partial}{\partial x_{j}} a^{j, k}(x) \frac{\partial}{\partial x_{k}} S(t, x, y) & =0, \quad t>0 \\
S(0, x, y) & =\delta(x-y) .
\end{aligned}
$$

Theorem CKS ([CKS, Theorem 1.9]). The point $(x, \xi, y, \eta) \in T^{*}\left(\mathbb{R}^{n}\right) \times$ $T^{*}\left(\mathbb{R}^{n}\right)$ does not belong to the wave front set of the fundamental solution $S(t, \cdot, \cdot)$ for all $t>0$ if either $(x, \xi)$ is not trapped backwards or $(y, \eta)$ is not trapped forwards.

The authors of $[\mathrm{CKS}]$ do not work with such initial data as $\delta(x-y)$. Instead, they work with the class of functions $f$ satisfying $(1+|x|)^{k} f(x) \in$ $L^{2}$, for all $k>0$. Thus, in order to prove their Theorem 1.9, the readers are instructed to approximate $\delta$-function by the functions $f$ of the above class, and check that all the intermediate results on the wave front sets of the solutions, all the estimates, survive passing to the limit.

Some time ago, not knowing of the research undertaken by Craig, Kappeler and Strauss, the authors of the present paper also obtained a result on dispersive smoothing for the Schrödinger equations with variable coefficients. Originally, this was done in the line of our work on the parametrix for the Schrödinger equations ([KS]; the work was reported by Yu. S. at the conference in Saint Jean de Monts, France, in the spring of 1990, and by L. K. at the Special Analysis Seminar at the Courant Institute in the fall of 1990). The result on the singularities of the fundamental solution 
that we needed and proved at that time was quite primitive compared to Theorem CKS. Namely, we showed that $S(t, x, y)$ has no singularities for $t>0$ provided all the points of the phase space $T^{*}\left(\mathbb{R}^{n}\right)$ are not trapped forwards.

Our approach is very different from that of [CKS]. Fortunately, we can handle some cases and situations not covered by the technique of [CKS]; for example, the initial boundary-value problems and systems of equations. Also, our method is simpler, and we believe that it provides an important additional insight into the basic properties of the Schrödinger equations.

The essence of our method is in exploiting the correspondence between the flows generated by the Schrödinger and hyperbolic equations and then using the "decay" of the local energy of the latter.

The correspondence between the flows is described by the following relation between the fundamental solutions of the Schrödinger and wave equations,

$$
\mathfrak{S}(t)=\frac{e^{-i \frac{\pi}{4}}}{\sqrt{4 \pi t}} \int e^{i \frac{\tau^{2}}{4 t}} \mathfrak{W}(\tau) d \tau, \quad t>0
$$

where $\mathfrak{S}(t)$ is the fundamental solution of the Schrödinger equation,

$$
\frac{1}{i} \frac{\partial}{\partial t} \mathfrak{S}(t)+A \mathfrak{S}(t)=0, \quad t>0, \quad \mathfrak{S}(0)=I,
$$

and $\mathfrak{W}(\tau)$ is the fundamental solution of the corresponding wave equation,

$$
\frac{\partial^{2}}{\partial \tau^{2}} \mathfrak{W}(\tau)+A \mathfrak{W}(\tau)=0, \quad \tau>0, \quad \mathfrak{W}(0)=I, \quad \frac{\partial}{\partial t} \mathfrak{W}(0)=0
$$

Here $A$ is an operator that in the simplest case will be a perturbed Laplacian, and $I$ is the identity operator.

An informal proof of (1) goes as follows. First, notice that the scalar function

$$
\mathbb{k}(t, \tau)=\frac{e^{-i \frac{\pi}{4}}}{\sqrt{4 \pi t}} e^{i \frac{\tau^{2}}{4 t}}
$$

is the fundamental solution of the 1-dimensional Schrödinger equation,

$$
\frac{1}{i} \frac{\partial}{\partial t} \mathbb{k}(t, \tau)-\frac{\partial^{2}}{\partial \tau^{2}} \mathbb{k}(t, \tau)=0, \quad t>0, \quad \mathbb{k}(0, \cdot)=\delta(\cdot)
$$


Therefore,

$$
\begin{aligned}
\left(\frac{1}{i} \frac{\partial}{\partial t}+A\right) \int \mathbb{k}(t, \tau) & \mathfrak{W}(\tau) d \tau \\
& =\int\left(\frac{1}{i} \frac{\partial}{\partial t} \mathbb{k}(t, \tau) \mathfrak{W}(\tau)+\mathbb{k}(t, \tau) A \mathfrak{W}(\tau)\right) d \tau \\
& =\int\left(\frac{\partial^{2}}{\partial \tau^{2}} \mathbb{k}(t, \tau) \mathfrak{W}(\tau)+\mathbb{k}(t, \tau) A \mathfrak{W}(\tau)\right) d \tau \\
& =\int \mathbb{k}(t, \tau)\left(\frac{\partial^{2}}{\partial \tau^{2}}+A\right) \mathfrak{W}(\tau) d \tau=0 .
\end{aligned}
$$

Hence, the right hand side of (1) does indeed represent the fundamental solution of the Schrödinger equation (2).

The argument showing that the fundamental solution $\mathfrak{S}(t)$ becomes regular for $t>0$ is based on the following observation:

$$
\begin{aligned}
t^{2} A \mathfrak{S}(t) & =t^{2} A \int \mathbb{k}(t, \tau) \mathfrak{W}(\tau) d \tau=t^{2} \int \mathbb{k}(t, \tau) A \mathfrak{W}(\tau) d \tau \\
& =t^{2} \int \mathbb{k}(t, \tau) \cdot \frac{d^{2}}{d \tau^{2}} \mathfrak{W}(\tau) d \tau=t^{2} \int \frac{\partial^{2}}{\partial \tau^{2}} \mathbb{k}(t, \tau) \cdot \mathfrak{W}(\tau) d \tau \\
& =i \frac{t}{2} \mathfrak{S}(t)-\frac{1}{4} \int \mathbb{k}(t, \tau) \tau^{2} \mathfrak{W}(\tau) d \tau
\end{aligned}
$$

In other words, multiplying by $t^{2}$ improves the regularity in spatial directions. Since $\frac{1}{i} \frac{\partial}{\partial t} \mathfrak{S}(t)=-A \mathfrak{S}(t)$, it improves the regularity in $t$ as well. Of course, we will have to justify the above manipulations, and, in particular, the fact that the regularity of $\int \mathbb{k}(t, \tau) \tau^{2} \mathfrak{W}(\tau) d \tau$ is the same as that of $\int \mathbb{k}(t, \tau) \mathfrak{W}(\tau) d \tau$.

In what follows, we first justify (1) in an abstract setting, and then show how it works in applications.

The existence, uniqueness and regularity properties of solutions for the Schrödinger equation will be viewed against the backdrop of a scale of Hilbert spaces $H_{s}, s \in \mathbb{R}$. We will also use the sets $H_{s}^{\text {comp }}$ of elements of $H_{s}$ with compact support, and the space $H_{s}^{\text {loc }}$, the "dual" of $H_{-s}^{\text {comp }}$. (One may think of $H_{s}$ as the usual Sobolev space $H^{s}\left(\mathbb{R}^{n}\right)$, then $H_{s}^{\text {comp }}$ and $H_{s}^{\text {loc }}$ have the natural meaning.) In order to localize the elements of $H_{s}^{\text {loc }}$ we use the "cut-off functions". Let $\Upsilon$ denote the set of cut-off functions or operators of multiplication by such functions (in practice, $\Upsilon \approx C_{0}^{\infty}\left(\mathbb{R}^{n}\right)$ ). 
Naturally, we assume that for every $f \in H_{s}^{\text {comp }}$ there is $\zeta \in \Upsilon$ such that $f=\zeta f$.

The operator $A$ will be thought of as a (second order) linear operator that maps $H_{s}$ continuously into $H_{s-2}$ for any $s \in \mathbb{R}$. We make an addi-

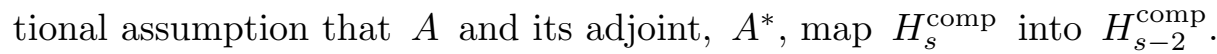

We will assume that the hyperbolic problem has a solution $\mathfrak{W}(t)$ in the following sense. First, $\frac{d^{\ell}}{d t^{\ell}} \mathfrak{W} \in C\left(\left[0, t_{1}\right] \rightarrow \operatorname{Hom}\left(H_{s}, H_{s-\ell}\right)\right)$, for all $t_{1}>0$, $\ell=0,1, \ldots$, and for every $s \in \mathbb{R}$. Here $\operatorname{Hom}\left(H_{s}, H_{s-\ell}\right)$ is the space of bounded linear operators from $H_{s}$ into $H_{s-\ell}$, and the continuity with respect to $t$ is understood in the strong operator topology. Second, given $f \in H_{s}$, the function $w(t)=\mathfrak{W}(t) f$ satisfies $w(0)=f, w_{t}(0)=0$, and $\left(w_{t t}(t)+A w(t), \eta\right)=0$, for all $\eta \in H_{-s+2}$.

We also have to make some assumptions on the behavior of $\mathfrak{W}(\tau)$ for large $|\tau|$. In applications, $\mathfrak{W}(\tau)$ will be the solving operator corresponding to an initial boundary-value problem for a single hyperbolic differential equation or a hyperbolic system. Not much can be said about the longterm behavior of $\mathfrak{W}(\tau)$ in the general case. However, if the so-called nontrapping condition is satisfied, then the local structure of $\mathfrak{W}(\tau)$ for large $|\tau|$ is known in considerable detail. By "local structure" we mean the structure of the operators $\zeta_{2} \cdot \mathfrak{W}(\tau) \cdot \zeta_{1}$ with arbitrary smooth, compactly supported functions $\zeta_{1}(\cdot)$ and $\zeta_{2}(\cdot)$ of spatial variables $x$. In our abstract setting, we make the assumptions on $\mathfrak{W}(\tau)$ that are in complete analogy with the most general known results on the long-term behavior of solutions of hyperbolic problems (with non-trapping condition), see [V 4], Theorems 10.3.4 and 10.3.6, and [Rau 1], Theorem 3.

Hypothesis $(\star)$. For any pair of cut-off functions $\zeta_{1}, \zeta_{2} \in \Upsilon$, there exists $T>1$ such that for $\tau \geq T-1$ the operator $\zeta_{2} \cdot \mathfrak{W}(\tau) \cdot \zeta_{1}$ can be written in the following form:

$$
\zeta_{2} \cdot \mathfrak{W}(\tau) \cdot \zeta_{1}=\sum_{j=1}^{N-1} \phi_{j}(\tau) e^{-i \lambda_{j} \tau} W_{j}+\tilde{W}_{N}(\tau),
$$

where the $\lambda_{j}$ 's are complex numbers with nonnegative imaginary parts, the scalar functions $\phi_{j}(z)$ are holomorphic in the half-plane $\operatorname{Re} z>T-1$ and satisfy there the following condition: for every finite interval $[a, b]$ there exists an integer $\ell_{j} \geq 0$ such that

$$
\sup _{a \leq \theta \leq b} \frac{1}{\tau^{\ell}} \sum_{m=0}^{\ell}\left|\frac{d^{m}}{d \tau^{m}} \phi_{j}(\tau+i \theta)\right|=O\left(\tau^{-2}\right) \quad \text { as } \quad \tau \rightarrow \infty, \quad \forall \ell \geq \ell_{j}
$$


The operators $W_{1}, \ldots, W_{N-1}$ do not depend on $\tau$ and are smoothing in the sense that each $W_{j}$ is bounded from $H_{s_{1}}$ to $H_{s_{2}}$ for any pair of $s_{1}, s_{2} \in \mathbb{R}$.

Finally, the operator $\tilde{W}_{N}(\tau)$ and all the derivatives $\frac{d^{\ell}}{d \tau^{\ell}} \tilde{W}_{N}(\tau)$, are smoothing for all $\tau \geq T-1$. Moreover, for any $M>0$ there is an integer $\kappa_{M} \geq 0$ such that, given $s_{1}, s_{2} \in \mathbb{R}$,

$$
\begin{aligned}
& \left\|\frac{d^{\ell}}{d \tau^{\ell}} \tilde{W}_{N}(\tau)\right\|_{H_{s_{1}} \rightarrow H_{s_{2}}}=O\left(\tau^{-M}\right), \quad \text { as } \quad \tau \rightarrow \infty, \\
& \ell=\kappa_{M}, \kappa_{M}+1, \ldots,
\end{aligned}
$$

where $\|\cdot\|_{H_{s_{1}} \rightarrow H_{s_{2}}}$ denotes the norm of an operator from $H_{s_{1}}$ to $H_{s_{2}}$.

Several situations where Hypothesis $(\star)$ is valid will be described later.

Theorem 1. Under the above assumptions on $\mathfrak{W}(t)$, there exists $\mathfrak{S}(t)$ that solves (2) in the following sense. First,

$$
\frac{d^{\ell}}{d t^{\ell}} \mathfrak{S} \in C\left(\left[0, t_{1}\right] \rightarrow \operatorname{Hom}\left(H_{s}^{\mathrm{comp}}, H_{s-2 \ell}^{\mathrm{loc}}\right)\right), \quad \forall t_{1}>0, \ell=0,1, \ldots,
$$

for every $s \in \mathbb{R}$. Second, given $f \in H_{s}^{\text {comp }}$, the function $u(t)=\mathfrak{S}(t) f$ satisfies $u(0)=f$ and $\left(\frac{1}{i} \frac{\partial}{\partial t} u(t)+A u(t), \eta\right)=0$, for all $\eta \in H_{-s+2}^{\mathrm{comp}}$ and all $t>0$.

The operator $\mathfrak{S}(t)$ has the following smoothing property:

$$
\left\{\begin{array}{l}
\text { for every integer } k>0, \\
\text { the operator-valued function } t \mapsto t^{2 k} A^{k} \mathfrak{S}(t) \text { is continuous in } t \\
\text { with values in } \operatorname{Hom}\left(H_{s}^{\mathrm{comp}}, H_{s}^{\mathrm{loc}}\right) .
\end{array}\right.
$$

The operator $\mathfrak{S}(t)$ can be expressed in terms of the operator $\mathfrak{W}$ as follows. For arbitrary $\zeta_{1}, \zeta_{2} \in \Upsilon$, there exists $T>1$ so that for any $C^{\infty}$ function $\vartheta_{0}(\tau)$ on $\mathbb{R}$ that equals 1 for $|\tau| \leq T$ and 0 for $|\tau| \geq T+1$, there exists a smoothing operator $\mathfrak{C}(t)$ (with infinitely smooth Schwartz kernel $\mathfrak{C}(t, x, y))$ such that

$$
\zeta_{1} \cdot \mathfrak{S}(t) \cdot \zeta_{2}=\int \mathbb{k}(t, \tau) \vartheta_{0}(\tau) \zeta_{1} \cdot \mathfrak{W}(\tau) \cdot \zeta_{2} d \tau+\mathfrak{C}(t)
$$

Sketch of the proof. We regularize integral (1) by inserting an extra factor $e^{-\varepsilon \tau^{2}}, \varepsilon>0$, into the integrand and then letting $\varepsilon \rightarrow 0$. Denote

$$
\mathfrak{S}_{\varepsilon}(t)=\int \mathbb{k}(t, \tau) e^{-\varepsilon \tau^{2}} \mathfrak{W}(\tau) d \tau .
$$


Our plan is to show first that, given $f \in H_{s}^{\text {comp }}$, the limit

$$
\lim _{\varepsilon \downarrow 0}\left(\mathfrak{S}_{\varepsilon}(t) f, \eta\right)=(\mathfrak{S}(t) f, \eta)
$$

exists for every $\eta \in H_{-s}^{\text {comp }}$ and correctly defines an operator

$$
\mathfrak{S}(t): H_{s}^{\mathrm{comp}} \rightarrow H_{s}^{\mathrm{loc}}
$$

Second, we have to check that $\psi(t)=\mathfrak{S}(t) f$ is a distributional solution of the problem $\frac{1}{i} \frac{\partial}{\partial t} \psi+A \psi=0, \psi(0)=f$. Finally, we have to justify (6) and (7).

The first step is the most involved. We pick two arbitrary cut-off functions $\zeta_{1,2} \in \Upsilon$. By Hypothesis $(\star)$, there is $T>1$ so that (5a) holds for $t \geq T-1$. Let $\vartheta_{0}(\tau)$ be a smooth real function that equals 1 for $\tau \leq T$ and 0 for $\tau \geq T+1$. Noting that $\mathfrak{W}(\tau)$ is an even function of $\tau$, we write

$$
\begin{gathered}
\zeta_{1} \cdot \mathfrak{S}_{\varepsilon}(t) \cdot \zeta_{2}=2 \int_{0}^{\infty} \mathbb{k}(t, \tau) e^{-\varepsilon \tau^{2}} \vartheta_{0}(\tau) \zeta_{1} \cdot \mathfrak{W}(\tau) \cdot \zeta_{2} d \tau \\
+2 \int_{0}^{\infty} \mathbb{k}(t, \tau) e^{-\varepsilon \tau^{2}}\left(1-\vartheta_{0}(\tau)\right) \zeta_{1} \cdot \mathfrak{W}(\tau) \cdot \zeta_{2} d \tau=\mathfrak{S}_{\varepsilon}^{(0)}(t)+\mathfrak{S}_{\varepsilon}^{(1)}(t) .
\end{gathered}
$$

The integral $\mathfrak{S}_{\varepsilon}^{(0)}(t)$ converges to $\mathfrak{S}^{(0)}(t)=2 \int_{0}^{\infty} \mathbb{k}(t, \tau) \vartheta_{0}(\tau) \zeta_{1} \cdot \mathfrak{W}(\tau) \cdot \zeta_{2} d \tau$ in the strong operator topology in any of the spaces $H_{s}$, as is obvious.

To treat $\mathfrak{S}_{\varepsilon}^{(1)}(t)$, we use $(5)$. The part of $\mathfrak{S}_{\varepsilon}^{(1)}(t)$ corresponding to $\tilde{W}_{N}$ in (5a) can be regularized by repeatedly using the equality

$$
\exp \left\{\left(\frac{i}{4 t}-\varepsilon\right) \tau^{2}\right\}=\left\{2 \tau\left(\frac{i}{4 t}-\varepsilon\right)\right\}^{-1} \frac{\partial}{\partial \tau} \exp \left\{\left(\frac{i}{4 t}-\varepsilon\right) \tau^{2}\right\}
$$

and integration by parts. The other terms in (5a) lead to the integrals of the form

$$
\int_{0}^{\infty} \mathbb{k}(t, \tau) e^{-\varepsilon \tau^{2}} e^{-i \lambda_{j} \tau} \phi_{j}(\tau)\left(1-\vartheta_{0}(\tau)\right) d \tau W_{j},
$$

where $\operatorname{Im} \lambda_{j} \geq 0$. We treat these integrals essentially in the same fashion as textbooks handle the convergence of $\int_{-\infty}^{+\infty} e^{i \tau^{2}} d \tau$ (by moving the contour, etc.). Again, integration by parts and use of ( $5 \mathrm{~b})$ take care of regularization. 
The same technique works to show that, given $f \in H_{s}^{\text {com }}$,

$$
\lim _{\varepsilon \searrow 0} \int_{0}^{\infty}\left(\mathfrak{S}_{\varepsilon}(t) f,\left(\frac{1}{i} \frac{\partial}{\partial t}+A^{*}\right) \eta(t)\right) d t=\frac{1}{i}(f, \eta(0)),
$$

for all $\eta \in C_{0}^{\infty}\left(\mathbb{R} ; H_{-s+2}^{\text {com }}\right)$ that vanish for large $t$.

The smoothing property is proved following the computation (4b) except for that we start with $t^{2} A \mathfrak{S}_{\varepsilon}(t)$ and then at the final step we let $\varepsilon \searrow 0$. We skip the details.

Remark. Note that we make no assumption that the operator $A$ be selfadjoint. It is known, however, that if $A$ is not self-adjoint, the initial value problem (2) may turn out to be ill-posed, at least in the $L^{2}$-based Sobolev spaces, see $[\mathrm{Tak}],[\mathrm{Mi}]$, and $[\mathrm{Ich}]$, for the discussion of necessary and of sufficient conditions for well-posedness. Moreover, if $A$ differs from $A^{*}$ by an operator of order $\geq 1$, then, in general, there are no energy estimates available for problem (2), and already the existence of solutions becomes problematic. Nonetheless, our Theorem 1 provides a construction for the solutions corresponding to the initial data of compact support.

Let us now turn to examples. The Hypothesis $(\star)$ is evidently the most restrictive ingredient of our approach. That hypothesis is motivated by the uniform local energy decay theorems for the wave equation in "nontrapping" exterior domains. The systematic study of that phenomenon has been initiated by C. S. Morawetz, and P. D. Lax and R. S. Phillips, and taken up by many other mathematicians (see, e.g., [Mo], [LP], [LMP], [Ral], [V], [S], [Rau 1], [Tay], [Me], [MS]). In the situations that were studied first, Hypothesis $(\star)$ holds with functions $\phi_{j}$ and exponents $k_{j} \in \mathbb{C}$ such that all $\left|D_{\tau}^{\ell} \phi_{j}(\tau) e^{-i k_{j} \tau}\right|$ do actually decay as $\tau \rightarrow+\infty$. Consider the following example.

Let $\Omega \subset \mathbb{R}^{n}, n \geq 2$, be a non-compact connected domain with compact, $C^{\infty}$ boundary $\partial \Omega$. Let $S(t, x, y)$ be the Schwartz kernel of the solution operator $S(t): f(\cdot) \mapsto u(t, \cdot)$ corresponding to the initial boundary-value problem

$$
\begin{aligned}
\frac{1}{i} \frac{\partial}{\partial t} u(t, x)-\Delta u(t, x) & =0, & & t \in \mathbb{R}, x \in \Omega, \\
\mathcal{B} u(t, x) & =0, & & x \in \partial \Omega, \\
u(0, x) & =f(x), & & x \in \Omega,
\end{aligned}
$$


where $\mathcal{B}$ is the boundary operator corresponding to either the Dirichlet or Neumann boundary condition.

Corollary 2. Assume that $\Omega$ is non-trapping in the sense that for every sufficiently large $R>0$ there exists $T_{R}>0$ such that no generalized geodesic of length $T \geq T_{R}$ lies completely within the ball $\{|x| \leq R\}$. (The generalized geodesics are the projections into $\Omega$ of the generalized bicharacteristics, see [MS 2], Definition 3.1.) Then the fundamental solution $S(t, x, y)$ of problem (8) is $C^{\infty}$ in $(0, \infty) \times \bar{\Omega} \times \bar{\Omega}$.

Proof. We choose the scale of Hilbert spaces generated from $H_{0}=L^{2}(\Omega)$ by the powers of the square root of the operator $A$, which, in our case, is $(-\Delta)$ with the boundary condition $\mathcal{B}$. The set $\Upsilon$ is comprised of the restrictions to $\Omega$ of the functions from $C_{0}^{\infty}\left(\mathbb{R}^{n}\right)$. That Hypothesis $(\star)$ is true follows from [Me], [Ral 2], and [V 3]. In particular, the functions $\phi_{j}(\tau), j=1, \ldots, N-1$, in (5a) can be omitted, i.e., $N=1$. Given $s_{1}, s_{2}$, the $H_{s_{1}} \rightarrow H_{s_{2}}$-norm of $\frac{d^{\ell}}{d \tau^{\ell}} \tilde{W}_{1}(\tau)$ behaves like $\frac{d^{\ell}}{d \tau^{\ell}} \tilde{\phi}_{1}(\tau)$ for large $\tau$, where $\tilde{\phi}_{1}(\tau)=e^{-r \tau}$ with $r>0$, in the case of odd $n$, and $\tilde{\phi}_{1}(\tau)=\tau^{-n}$, if $n$ is even.

Our next example is the Schrödinger equation with variable coefficients in $\mathbb{R}^{n}, n \geq 1$. Let

$$
A=-a^{j, k}(x) \frac{\partial^{2}}{\partial x_{j} \partial x_{k}}+b^{j}(x) \frac{\partial}{\partial x_{j}}+b^{0}(x),
$$

with summation over the repeated indices assumed. The coefficients $a^{j, k}$, $b^{j}$ and $b^{0}$ are supposed to be infinitely differentiable, the matrix $\left\{a^{j, k}(x)\right\}$ is real, symmetric and positive definite, uniformly with respect to $x \in \mathbb{R}^{n}$. The coefficients $b^{j}$ and $b^{0}$ are, in general, complex valued. In addition, we assume that $a^{j, k}, b^{j}$ and $b^{0}$ stabilize outside some ball:

$$
a^{j, k}(x)=a_{\infty}^{j k}, \quad b^{j}(x)=b_{\infty}^{j}, \quad b^{0}(x)=b_{\infty}^{0}, \quad \text { for } \quad|x| \geq R .
$$

Let $(q(s ; x, \xi), p(s ; x, \xi))$ be the Hamiltonian trajectory corresponding to the Hamilton function $H(q, p)=a^{j, k}(q) p_{j} p_{k}$ and starting at $q(0 ; x, \xi)=x$, $p(0 ; x, \xi)=\xi$.

Corollary 3. Assume that $|q(s ; x, \xi)| \rightarrow \infty$ as $s \rightarrow+\infty$, for all $x \in$ $\mathbb{R}^{n}$ and $\xi \in \mathbb{R}^{n} \backslash 0$ (the non-trapping condition). Then there exists a 
one-parameter family $S_{A}(t), 0<t<+\infty$, of operators $S_{A}(t): \mathcal{E}^{\prime}\left(\mathbb{R}^{n}\right) \rightarrow$ $C^{\infty}\left(\mathbb{R}^{n}\right)$ that solves the initial-value problem

$$
\frac{1}{i} \frac{\partial}{\partial t} \psi+A \psi=0,\left.\quad \psi\right|_{t=0}=f
$$

for all $f \in \mathcal{E}^{\prime}\left(\mathbb{R}^{n}\right)$, i.e., $\psi(t, x)=\left(S_{A}(t) f\right)(x)$ is a solution of $(9 \mathrm{c})$. (Here $\mathcal{E}^{\prime}\left(\mathbb{R}^{n}\right)$ is the space of distributions of compact support on $\mathbb{R}^{n}$.)

Proof. If necessary, we can change the independent variables $x \rightarrow y=$ $M x$ to set $M a_{\infty} M^{\prime}=\operatorname{diag}\{1, \ldots, 1\}$, and seek the solution of (9c) in the form $\psi(t, x)=\exp \left\{\omega \cdot y-i \omega^{0} t\right\} \phi(t, y)$. Choosing $\omega=-\frac{1}{2} M b_{\infty}$ and $\omega^{0}=b_{\infty}^{0}-\frac{3}{4} M b_{\infty} \cdot M b_{\infty}$, we get for $\phi(t, y)$ a similar problem but with an elliptic operator stabilizing to $-\Delta$ outside some ball $\left\{|y| \leq R^{\prime}\right\}$. Thus, from the very beginning we may assume that $a_{\infty}^{j k}=\delta^{j k}$ and $b_{\infty}^{0}=b_{\infty}^{1}=$ $\cdots=b_{\infty}^{n}=0$.

Next, we use Theorem 1 with the cut-off functions from $\Upsilon=C_{0}^{\infty}\left(\mathbb{R}^{n}\right)$. Because of our non-trapping assumption, the corresponding version of $\mathrm{Hy}-$ pothesis $(\star)$ holds true. Again, the functions $\phi_{j}(\tau), j=1, \ldots, N-1$, in (5a) can be omitted, and we have $\tilde{\phi}_{1}(\tau)=e^{-r \tau}$ with $r>0$, if $n$ is odd, and $\tilde{\phi}_{1}(\tau)=\tau^{-n}$, if $n$ is even. This follows from [V 4], chapters 9 and 10, and [Rau 1]; for the case of the formally selfadjoint $A$ see also [PoS].

A generalization to systems of Schrödinger equations is straightforward. Consider, for example, the following version of the Schrödinger evolution in a locally curved space with a gauge magnetic field.

Let $\mathbf{g}=g_{j k}(x) d x_{j} d x_{k}$ be a smooth Riemannian metric in $\mathbb{R}^{n}$, flat outside a ball: $g_{j k}(x)=\delta_{j k}$, if $|x| \geq R>0$. Let $A_{1}(x), \ldots, A_{n}(x)$ be a given $n$-tuple of smooth functions of $x$ with values in the Lie algebra $g \ell(m, \mathbb{C})$ of all complex $m \times m$-matrices, $m \geq 1$. We assume that $A_{1}(x), \ldots, A_{n}(x)$ are constant outside of the ball $|x| \leq R\}$ and satisfy there the following commutator condition:

$$
\left[A_{j}+A_{j}^{\dagger}, A_{k}+A_{k}^{\dagger}\right]=0, \quad \forall j, k,
$$

where $A_{j}^{\dagger}$ is the Hermite conjugate of $A_{j}$.

The Schrödinger field will be represented by a column vector $\vec{\psi}(t, x) \in$ $\mathbb{C}^{m}$. We denote $\nabla_{j}=i \frac{\partial}{\partial x_{j}}+A_{j}(x)$, the $j$-th covariant derivative, and $\nabla_{j}^{*}$, its conjugate with respect to the $L^{2}$-product

$$
(\vec{\xi}, \vec{\eta})=\int g^{j k}(x) \xi_{j}(x) \overline{\eta_{k}(x)} \sqrt{g} d x
$$


Finally, denote

$$
A=\frac{1}{2} \sum_{j=1}^{n}\left(\nabla_{j}^{*} \nabla_{j}+\nabla_{j} \nabla_{j}^{*}\right),
$$

and consider the Cauchy problem

$$
\frac{1}{i} \frac{\partial}{\partial t} \vec{\psi}+A \vec{\psi}=0,\left.\quad \vec{\psi}\right|_{t=0}=\vec{\psi}^{0}
$$

Corollary 3. If the metric $\mathbf{g}$ has no trapped geodesics then the (matrix valued) fundamental solution $S_{\frac{1}{i} \frac{\partial}{\partial t}+A}(t, x, y)$, corresponding to (10c), is infinitely differentiable for $t>0, x, y \in \mathbb{R}^{n}$.

Remark 4. In many cases, including those considered above for $n \geq 3$, the localized solving operator for the hyperbolic problem, $\zeta_{2} \cdot \mathfrak{W}(\tau) \cdot \zeta_{1}$, does, in fact, decay as $\tau^{-3}$ when $\tau \rightarrow \infty$, which makes the justification of the abstract scheme, given in the opening paragraphs, quite trivial. Also, then we get a simpler representation for $\zeta_{2} \cdot \mathfrak{S}(t) \cdot \zeta_{1}$, namely,

$$
\zeta_{1} \cdot \mathfrak{S}(t) \cdot \zeta_{2}=\int \mathbb{k}(t, \tau) \zeta_{1} \cdot \mathfrak{W}(\tau) \cdot \zeta_{2} d \tau
$$

As a serendipitous consequence, we obtain the following time-decay result for the perturbed Schrödinger evolution:

$$
\left\|\zeta_{1} \cdot \mathfrak{S}(t) \cdot \zeta_{2}\right\|_{H_{s} \rightarrow H_{s}} \leq \frac{c\left(s, \zeta_{1}, \zeta_{2}\right)}{\sqrt{t}}
$$

for all $s$ and any pair of cut-off functions $\zeta_{1,2}$. In particular, if $n \geq 3$, then at least $1 / \sqrt{t}$-decay rate holds for each of the three examples considered above.

The time-decay for the solutions of $\frac{1}{i} \frac{\partial}{\partial t} \psi-\Delta \psi+V \psi=0$ has been studied by several authors for different classes of the potentials $V$, see [Ste], [Rau 2], [J], [JK], [Mu]. It has been shown, in particular, that the $1 / \sqrt{t}$-decay is generic, while for special classes of potentials the rate of decay may be higher. 


\section{Concluding remarks}

Thus we have shown that the simple relation (1) between the Schrödinger and wave evolutions allows us to prove the smoothness of the fundamental solution for the Schrödinger equation, and also, the local time decay. The method works in the cases where the local energy decay for the hyperbolic evolution is known. We think that (1), though transparent, is important. It should be mentioned that (1) was implicitly used in [BdeM] in the study of the propagation of singularities for equations with double characteristics.

Comparing our results with those of Craig, Kappeler and Strauss, [CKS], one notices that we avoid microlocal statements. The reason is that there is no microlocal version of the local energy decay for hyperbolic problems available.

Question. Under what conditions on the operator (9a) and the pseudodifferential operators $\zeta_{1,2}\left(x, D_{x}\right)$ does the operator $\zeta_{2} \cdot \mathfrak{W}(\tau) \cdot \zeta_{1}$ satisfy (5) for large $\tau$ ?

We conjecture that at least the following holds. Let $K \subset \mathbb{R}_{x}^{n}$ be a compact set and $\Gamma \subset \mathbb{R}_{\xi}^{n}$ be a cone. Let $A$ be a uniformly elliptic operator of the form (9a) with smooth coefficients that stabilize at infinity so that $A=-\Delta$ for $|x| \geq R>0$. Denote by $(q(s ; x, \xi), p(s ; x, \xi))$, as before, the Hamilton trajectories corresponding to $H(q, p)=a^{j, k}(q) p_{j} p_{k}$. We say that the conic set $K \times \Gamma$ is non-trapping if all the rays starting from the points in $K \times(\Gamma \backslash 0)$ go to infinity: $|q(t ; x, \xi)| \rightarrow \infty$ as $t \rightarrow \infty$.

Let $v(x, \xi)$ be a (full) symbol from $S^{0}\left(\mathbb{R}_{x}^{n} \times \mathbb{R}_{\xi}^{n}\right)$, which has support in the conic set $K \times \Gamma$. Denote $\zeta_{1}\left(x, D_{x}\right)=\operatorname{Op} v$. Let $\zeta_{2} \in C_{0}^{\infty}\left(\mathbb{R}^{n}\right)$. As before, $\mathfrak{W}(\tau): f \mapsto w(\tau)$ will be the solution operator of the hyperbolic problem $w_{\tau \tau}+A w=0, w(0)=f, w_{\tau}(0)=0$.

Conjecture. If $K \times \Gamma$ is non-trapping, then for all sufficiently large $\tau>0$ the operator $\zeta_{2} \cdot \mathfrak{W}(\tau) \cdot \zeta_{1}$ is smoothing and its $\operatorname{Hom}\left(H^{s_{1}}, H^{s_{2}}\right)$-norms decay as $\tau \rightarrow \infty$.

\section{Acknowledgement}

The authors are grateful to Walter Craig and Walter Strauss for providing the manuscript of the paper [CKS] and for sharing the enthusiasm about the Schrödinger equation. 


\section{References}

[BdeM] L. Boutet de Monvel, Propagation des singularités des solutions d'équations analogues à l'équa-tion de Schrödinger, Fourier Integral Operators and Partial Differential Equations, (Lecture Notes in Math., No. 459), Springer-Verlag, Berlin-Heidelberg-New York, 1975.

[CKS] W. Craig, T. Kappeler and W. Strauss, Microlocal dispersive smoothing for the Schrödinger equation, preprint (March 1994).

[CFKS] H. L. Cycon, R. G. Froese, W. Kirsch, and B. Simon, Schrödinger operators, Springer-Verlag, Berlin-Heidelberg, 1987.

[I 1] W. Ichinose, The Cauchy problem for Schrödinger type equations with variable coefficients, Osaka J. Math. 24 (1987), 853-886.

[I 2] — On $L^{2}$ well posedness of the Cauchy problem for Schrödinger type equations on the Riemannian manifold and the Maslov theory, Duke Math. J. 56 (1988), no. 3, 549-588.

[J 1] A. Jensen, Local decay in time of solutions to Schrödinger's equation witha dilation-analytic interaction, Manuscripta Math. 25 (1978), 61-77.

[J 2] Spectral properties of Schrödinger operators and time-decay of the wave functions. Results in $L^{2}\left(\mathbb{R}^{m}\right), m \geq 5$, Duke Math. J. 47 (1980), 57-80; Results in $L^{2}\left(\mathbb{R}^{4}\right)$ :, J. Math. Anal. Appl. 101 (1984), 397-422.

[JK] A. Jensen and T. Kato, Spectral properties of Schrödinger operators and timedecay of the wave functions, Duke Math. Journal 46 (1979), 583-611.

[KS] L. Kapitanski and Yu. G. Safarov, Global fundamental solution of the Schrödinger evolution equation, in preparation.

[Ki 1] H. Kitada, On a construction of the fundamental solution for Schrödinger equations, J. Fac. Sci. Univ. Tokyo IA 27 (1980), 193-226.

[Ki 2] _ Fundamental Solutions and eigenfunction expansions for Schrödinger operators: I. Fundamental solutions, Math. Z. 198 (1988), 181-190.

[LP] P. D. Lax and R. S. Phillips, Scattering theory, 1st edition: 1967, 2nd edition: 1989, Academic Press, New York-Boston.

[LMP] P. D. Lax, C. S. Morawetz, and R. S. Phillips, Exponential decay of solutions of the wave equation in exterior of a star-shaped obstacle, Comm. Pure Appl. Math. 16 (1963), 477-486.

[MF] V. P. Maslov and M. V. Fedoriuk, Semi-classical approximation in quantum mechanics, D. Reidel Publ. Co., Dordrecht, Holland, 1981.

[Me] R. B. Melrose, Singularities and energy decay in acoustical scattering, Duke Math. J. 46 (1979), no. 1, 43-59.

[MS] R. B. Melrose and J. Sjostrand, Singularities of boundary problems, I, Comm. Pure Appl. Math. 31 (1978), 593-617; II, Comm. Pure Appl. Math. 35 (1982), 129-168.

[Mi] S. Mizohata, On the Cauchy problem, Notes and reports in Mathematics in Science and Engineering, 3, Academic Press, Inc., Orlando, FL, and Science Press, Beijing, 1985. 
[Mo 1] C. S. Morawetz, The decay of solutions of the exterior initial-boundary value problem for the wave equation, Comm. Pure Appl. Math. 14 (1961), 561-568.

[Mo 2] _ The limiting amplitude principle, Comm. Pure Appl. Math. 15 (1962), 349-361.

[Mo 3] Exponential decay of solutions of the wave equation, Comm. Pure Appl. Math. 19 (1966), 439-444.

[Mo 4] - Time decay for the nonlinear Klein-Gordon equation, Proc. Roy. Soc. A. 306 (1968), 291-296.

[Mo 5] - Decay for solutions of the exterior problem for the wave equation, Comm. Pure Appl. Math. 28 (1975), 229-264.

[MRS] C. S. Morawetz, J. V. Ralston, and W. Strauss, Decay of solutions of the wave equation outside nontrapping obstacles, Comm. Pure Appl. Math. 30 (1977), 447-508.

[Mu M. Murata, Rate of decay of local energy and spectral properties of elliptic operators, Japan J. Math. 60 (1980), 77-127.

[OF] T. A. Osborn and Y. Fujiwara, The evolution kernels: uniform asymptotics expansions, J. Math. Phys. 24 (1983), no. 5, 1093-1103.

[PoS] G. S. Popov and M. A. Shubin, Asymptotic expansion of the spectral function for second-order elliptic operators in $\mathbb{R}^{n}$, Functional Anal. Appl. 17 (1983), no. 3, 193-200.

[Ral 1] J. Ralston, Solutions of the wave equation with localized energy, Comm. Pure Appl. Math. 22 (1969), 807-823.

[Ral 2] _ Note on the decay of acoustical waves, Duke Math. J. 46 (1979), 799804.

[Rau 1] J. Rauch, Asymptotic behavior of solutions to hyperbolic partial differential equations with zero speeds, Comm. Pure Appl. Math. 31 (1978), 431-480.

[Rau 2] _ Local decay of scattering solutions to Schrödinger's equation, Comm. Math. Phys. 61 (1978), 149-168.

[Ste] S. Steinberg, Local time decay for solutions of the Schrödinger equation and the wave equation, Archive for Rational Mech. Anal. 54 (1974), no. 2, 134-147.

[Str] W. A. Strauss, Dispersal of waves vanishing on the boundary of an exterior domain, Comm. Pure Appl. Math. 28 (1975), 265-278.

[Tak] J. Takeuchi, A necessary condition for the well-posedness of the Cauchy problem for a certain class of evolution equations, Proc. Japan Acad. Ser.A Math. Sci. 50 (1974), 133-137.

[Tay] M. Taylor, Grazing rays and reflection of singularities of solutions to wave equations, Comm. Pure and Appl. Math. 29 (1976), 1-38 and 463-481; II (systems), Comm. Pure and Appl. Math. 29 (1976), 463-481.

[V 1] B. R. Vainberg, Behavior of the solution of the Cauchy problem for a hyperbolic equation as $t \rightarrow \infty$, Math. USSR-Sbornik 7 (1969), no. 4, 533-567.

[V 2]_ On exterior elliptic problems polynomially depending on a spectral parameter, and the asymptotic behavior for large time of solutions of nonstationary problems, Math. USSR-Sbornik 21 (1973), no. 2, 221-239. 
[V 3] - On the short wave asymptotic behavior of solutions of stationary problems and the asymptotic behavior as $t \rightarrow \infty$ of solutions of non-stationary problems, Russian Math. Surveys 30 (1975), no. 2, 1-58.

[V 4] - Asymptotic methods in equations of mathematical physics, Moscow University Press, 1982 (Russian); English translation: Gordon and Breach Science Publishers, New York-London, 1989.

[We] A. Weinstein, A symbol class for some Schrödinger equations on $\mathbb{R}^{n}$, Amer. Journ. Math. 107 (1985), 1-23.

[Wi] C. Wilcox, The initial-boundary value problem for the wave equation in an exterior domain with spherical boundary, Amer. Math. Soc. Not., Abstract No.564206 (1959).

[Za] E. C. Zachmanoglou, The decay of solutions of the initial-boundary value problem for hyperbolic equations, J. Math. Anal. Appl. 13 (1966), 504-515.

[Ze] S. Zelditch, Reconstruction of singularities for solutions of Schrödinger equations, Comm. Math. Phys. 90 (1983), 1-26.

Department of Mathematics, Kansas State Univerity, Manhatan, K ANSAS 66506 , U S A

E-mail address: levkapit@math.ksu.edu

Department of Mathematics, King's College, London Univerity, Strand, L ONDON W C 2 R $2 \mathrm{LS}$, ENGLAND, U K

E-mail address: ysafarov@mth.kcl.ac.uk 